\title{
TENDÊNCIAS ANUAIS E SAZONAIS NOS EXTREMOS DE TEMPERATURA DO AR E PRECIPITAÇÃO EM CAMPINA GRANDE - PB
}

\section{LEYDSON GALVÍNCIO DANTAS ${ }^{1}$,CARLOS ANTONIO COSTA DOS SANTOS ${ }^{1}$,RICARDO ALVES DE OLINDA ${ }^{2}$}

\author{
'Universidade Federal de Campina Grande, Unidade Acadêmica de Ciências Atmosféricas, Campina \\ Grande, PB, Brasil \\ ${ }^{2}$ Universidade Estadual da Paraíba, Departamento de Estatística, Campina Grande, PB, Brasil \\ leydsongalvincio@gmail.com,carlostorm@gmail.com,carlos@dca.ufcg.edu.br
}

Recebido Outubro de 2013 - Aceito Fevereiro de 2015

\begin{abstract}
RESUMO
Devido à rápida expansão urbana e potencial turístico da região, o objetivo deste estudo foi a obtenção da distribuição temporal dos índices de extremos climáticos dependentes de dados diários de temperatura e precipitação, definido pela OMM, para o período de 1975 a 2011, e utilizando os testes estatísticos não paramétricos obter a magnitude das tendências dos referidos índices para a cidade de Campina Grande - PB. Os resultados mostram que as temperaturas mínimas e máxima diárias têm aumentado gradativamente ao longo das últimas décadas, apresentando tendências sazonais crescentes ao longo dos anos e que as temperaturas mínimas tiveram maior aumento. O número de dias e noites quentes por ano tem aumentado, proporcionando assim uma redução significativa dos dias e noites frias. Foi observado que as temperaturas mínimas e máximas apresentam maiores tendências de aumento nas estações do outono e primavera, respectivamente. Baseado nos índices extremos de precipitação, mesmo sem significância estatística, foi observado que as chuvas intensas tendem a aumentar, ocorrendo em um período menor de tempo.
\end{abstract}

Palavras-Chave: aquecimento global, IPCC, urbanização, semiárido

\begin{abstract}
TRENDS IN ANNUAL AND SEASONAL TEMPERATURE AND PRECIPITATION EXTREMES IN CAMPINA GRANDE - PB

Due to the rapid urban expansion and tourism potential of the region the objective of this study was to obtain the temporal distribution of extreme climatic indices dependent on daily temperature and precipitation which were defined by WMO for the period 1975-2011, and by using the non-parametric statistical tests to obtain the magnitude of the trends for the studied indices at the Campina Grande city - PB. The results showed that the minimum and maximum daily temperatures have increased gradually over the last decades, showing increased seasonal trends over the years and minimum temperatures experienced a greater trend increase. The number of warmer daytimes and nights per year has increased, thereby providing a significant reduction in colder daytimes and nights. It was observed that the minimum and maximum temperatures have higher trends of increase in the fall and spring seasons, respectively. Based on extreme precipitation indices, although not statistically significant, it was observed that heavy rains tend to increase, occurring in a shorter period of time.
\end{abstract}

Keywords: global warming, IPCC, urbanization, semi-arid 


\section{INTRODUÇÃO}

O aquecimento do sistema climático é inequívoco, e desde os anos 1950, muitas das mudanças observadas são sem precedentes ao longo de décadas e milênios. A atmosfera e o oceano se aqueceram, as quantidades de neve e gelo têm diminuído, o nível do mar subiu e as concentrações de gases de efeito estufa aumentaram. Além disso, cada uma das três últimas décadas tem sido sucessivamente mais quente na superfície da Terra do que qualquer década anterior, desde 1850 (IPCC, 2013). No Quinto Relatório de Avaliação do IPCC (Intergovernmental Panel on Climate Change) (IPCC, 2013) foi detectada a influência humana no aquecimento da atmosfera e em mudanças em alguns eventos climáticos extremos.

As possíveis mudanças climáticas poderão acarretar significativos impactos nos setores natural, social e econômico, assim como, os extremos do clima associados à temperatura e precipitação poderão também afetar o consumo de energia, conforto humano e turismo (Santos et al., 2009; Santos e Manzi, 2011). Kostopoulo e Jones (2005) afirmam que o aumento nas perdas econômicas devido a extremos de tempo (chuvas intensas, ondas de calor, frios intensos entre outros) e, especialmente, o aumento nas perdas de vidas, tem sido regularmente notícias de jornais, despertando o interesse da comunidade científica em estudar os fenômenos climáticos.

Estudos como o de Liebmann et al. (2004), desenvolvido para a região central da América do Sul, devido ser a região com maiores densidades populacional e de produtividade agrícola, identificou tendências lineares sazonais na precipitação durante 1976-1999, mostrando que ocorreu um aumento no percentual de dias chuvosos sobre a região. $\mathrm{O}$ estudo realizado por Santos e Brito (2007), utilizando a metodologia adotada por Haylock et al. (2006), identificou tendências de aumento da precipitação total anual para os estados do Rio Grande do Norte e Paraíba. Santos et al. (2009) e Santos e Manzi (2011) estudaram eventos extremos de precipitação no estado do Ceará e suas relações com a temperatura dos oceanos tropicais e os resultados mostraram que apenas a região norte do Ceará apresenta tendências com alta significância estatística para os índices de eventos extremos, existindo características de aumento nas intensidades das secas e diminuição dos eventos de precipitação forte. De acordo com Marengo et al. (2009), simulações elaborados para dois cenários de emissões de gases de efeito estufa mostram que uma consequência do aquecimento global é uma intensificação do ciclo hidrológico. Na região Nordeste do Brasil essas mudanças podem variar de 1 a 4 ${ }^{\circ} \mathrm{C}$ de aquecimento com reduções de precipitação entre $10 \mathrm{e}$ $15 \%$, e que no futuro, essas tendências seriam vistas através de um aumento nas tendências dos dias consecutivos secos, acompanhados por reduções dos eventos de precipitação intensa.
Análises das temperaturas em muitas regiões do globo têm mostrado algumas importantes mudanças nos extremos. A temperatura global média da superfície tem experimentado significante aumento nos últimos 50 anos (Folland et al., 2001). De acordo com o Climate Research Unit da Universidade de East Anglia a temperatura global do ar durante o período de 2001 a 2010 foi $0,22^{\circ} \mathrm{C}$ mais quente do que a década de 1991 a 2000 (Jones, 2013). No entanto, para o NEB ainda existem poucos estudos publicados sobre mudanças na temperatura do ar e seus extremos. Vincent et al. (2005) observaram tendências nos índices extremos de temperatura sobre a América do Sul utilizando uma base de dados de 1960 a 2000 e identificaram que os extremos de temperatura estão mudando na América do Sul. Os autores não encontraram mudanças consistentes nos índices baseados nas temperaturas máximas diárias, mas tendências significativas foram observadas nos índices baseados nas temperaturas mínimas diárias. Esses resultados estão de acordo com o que tem sido observado em diferentes regiões do globo.

Eventos extremos de precipitação e temperatura estão bem documentados nas escalas global, continental e regional, no entanto, na escala local existem poucos estudos desenvolvidos. Sanderson et al. (2011) evidenciam que muitas partes isoladas dos continentes apresentam aquecimentos até três vezes mais rápido do que a média global. Entre os muitos fatores que podem estar associados a esse aquecimento se destaca a mudança do uso do solo, que ocorre de forma acelerada nas cidades em desenvolvimento e nas áreas em processo de desmatamento. Esses fatos justificam a necessidade de se estudar os eventos extremos climáticos dependentes da precipitação e temperatura diários para cidades em desenvolvimento, que passam por mudanças aceleradas do uso do solo, as quais podem aumentar a vulnerabilidade criando potenciais impactos nos eventos climáticos extremos. Dessa forma, Boccolari e Malmusi (2013) investigaram o comportamento do clima na área de Modena na Itália e verificaram que no período entre 1970 e 2010, as temperaturas máxima e mínima tiveram tendências de aumento e que, respectivamente, no verão e inverno essas tendências foram de aproximadamente $1{ }^{\circ} \mathrm{C}$, enquanto que a porcentagem de dias e noites frias está diminuindo, quase na mesma proporção em que os dias e noites quentes aumentam. Resultado similar foi encontrado por Chan et al. (2012), que observaram tendências de aumento nas temperaturas mínima, média e máxima, na cidade de Hong Kong na China, acarretando no decréscimo dos dias e noites frias e aumento de dias e noites quentes com tendências sazonais e anuais significativas, justificando que a possibilidade da causa do aumento da temperatura foi por conta da urbanização.

Campina Grande é o segundo município em população do estado da Paraíba. Sua área territorial é de 594,182 km² e exerce grande influência política e econômica no estado. A população de Campina Grande foi estimada em 400.002 
habitantes, correspondendo a uma densidade demográfica de $648,31 \mathrm{hab} / \mathrm{km}^{2}$ (IBGE, 2014). Medeiros (2011), através do uso da normal padronizada, identificou que nas duas últimas décadas, a temperatura média de Campina Grande se comportou acima das normais climatológicas, observando que houve uma elevação da temperatura média máxima, com maior amplitude de desvio em torno de $2,4^{\circ} \mathrm{C}$ para o mês de dezembro, e que em relação à temperatura média mínima, o menor desvio encontrado foi de $0,6{ }^{\circ} \mathrm{C}$ observado no mês de agosto. Os dados analisados mostraram temperaturas médias mensais que variaram de 0,4 ${ }^{\circ} \mathrm{C}$ a $2,0^{\circ} \mathrm{C}$ em relação às normais climatológicas oficiais e que demonstram uma grande variabilidade da temperatura nos últimos 20 anos e que podem estar relacionadas diretamente ao processo de urbanização da cidade.

Ao estudar a variabilidade da precipitação em Campina Grande, por análise dos coeficientes de correlação, Diniz (2013) observou que durante todo o ciclo anual, as maiores médias mensais de precipitação são verificadas entre os meses de maio e julho, com o máximo ocorrendo no mês de junho. Após esse período inicia-se uma progressiva diminuição das chuvas, alcançando valores mínimos nos três últimos meses do ano. Verificou-se, também, que o coeficiente de variação do número de dias com chuvas é inversamente proporcional ao total de precipitação. Além disso, as correlações entre o número de dias com chuvas e os totais mensais e anuais de precipitação são significativas.

A cidade de Campina Grande não foi estruturalmente planejada para o futuro, a mesma tem crescido de forma rápida e desordenada nestas últimas décadas, conforme pode ser observado na Figura 1. Cunha et al. (2009), baseados em análise de índices de vegetação, notaram diminuição das áreas verdes ao longo do processo de ocupação da cidade. A arborização urbana em Campina Grande é escassa por ter sido implantada deliberadamente sem planejamento voltado para o futuro, para o desenvolvimento e o crescimento da cidade (Carneiro, 2008). Assim, devido à sua rápida expansão urbana (Figuras 1b e 1c) e seus possíveis efeitos no clima local, torna-se necessário obter a distribuição temporal dos índices de extremos climáticos dependentes da temperatura e precipitação diárias para a cidade de Campina Grande, que apresenta uma série homogênea de dados, verificando os sinais das tendências temporais, utilizando o teste de Mann-Kendall para os níveis de significância adotados, e pelo teste não paramétrico de Sen (Sen-Slope) estimar a magnitude das tendências dos referidos índices. Além disso, não há estudos desenvolvidos para a referida cidade que aborde a temática de extremos climáticos analisando, conjuntamente, dados diários de precipitação e temperaturas máxima e mínima. a)

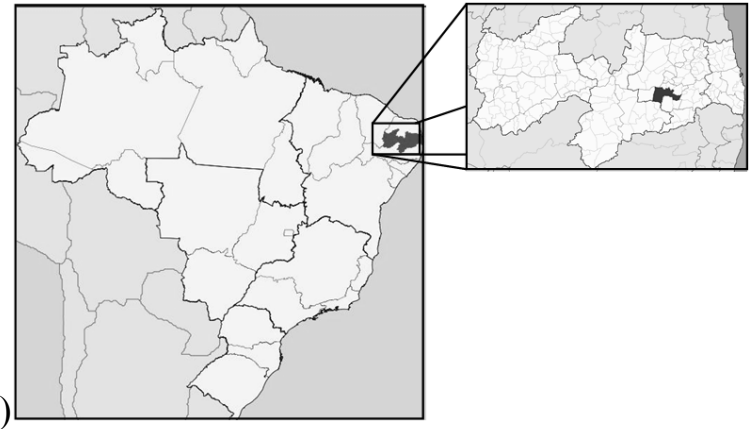

b)

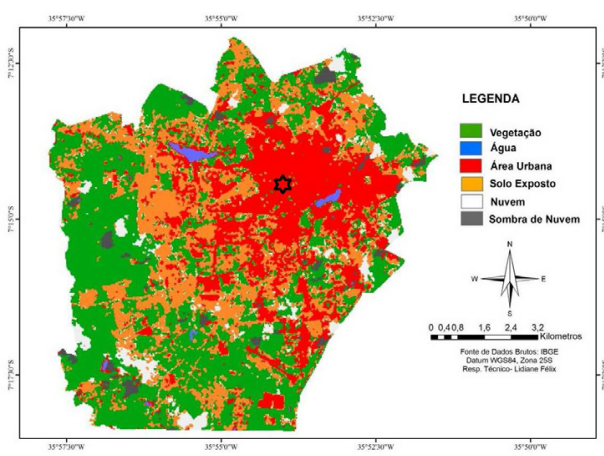

c)

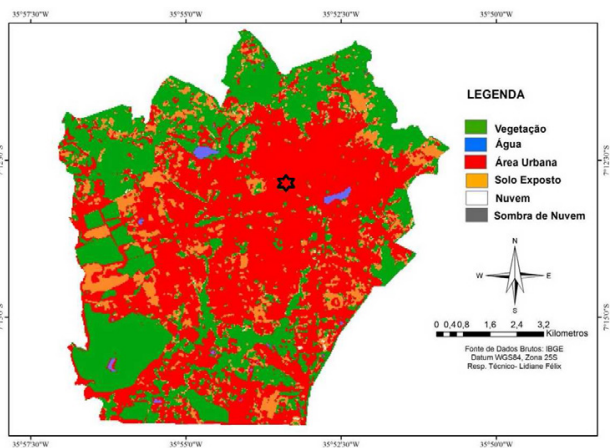

Figura 1 - a) Localização do estado da Paraíba no mapa do Brasil com destaque para o município de Campina Grande e malha urbana de Campina Grande para os anos de b) 1984 e c) 2011, com destaque para a localização da estação meteorológica do INMET. O símbolo da estrela (cor preta) corresponde à localização da estação meteorológica. 


\section{MATERIAL E MÉTODOS}

\section{1 Área de estudo e dados}

O município de Campina Grande está localizado no estado da Paraíba, na região oriental do Planalto da Borborema, conforme descrito na Figura 1. Na referida figura, tem-se a localização do estado da Paraíba no mapa do Brasil, com destaque para o município de Campina Grande e malha urbana de Campina Grande, obtida por imagens do satélite Landsat 5-TM, para os anos de 1984 e 2011, com destaque para a localização da estação meteorológica do Instituto Nacional de Meteorologia (INMET). O município apresenta um relevo variando de $337 \mathrm{~m}$ e $665 \mathrm{~m}$ acima do nível médio do mar (Medeiros, 2011). Utilizou-se 37 anos (1975 a 2011) de dados diários da temperatura do ar (máxima e mínima) e precipitação, proveniente da estação meteorológica do INMET instalada na sede da Empresa Brasileira de Pesquisa Agropecuária (EMBRAPA - Algodão) com coordenadas geográficas de $7^{\circ} 13$ '11' de latitude Sul e 35'52'31' de longitude Oeste, localizada na zona urbana (Figuras 1b e 1c) de Campina Grande - PB. A referida estação foi escolhida por apresentar séries de dados consistentes e com poucas falhas, apenas de ordem técnica (por exemplo, substituição de sensores), não impactando de forma considerável a série histórica. Além de não ter sido deslocada nem sofrida modificações antrópicas em seu entorno ao longo do período de estudo e por estar localizada nas proximidades do centro da cidade (Figuras $1 \mathrm{~b}$ e 1c).

\subsection{Metodologia}

Os índices de eventos extremos climáticos, propostos pelo ETCCDMI da OMM, aplicado a estudos locais, foram calculados através do software RClimdex, desenvolvido por Xuebin Zhang e Feng Yang para o Serviço Meteorológico Canadense (Zhang e Yang, 2004), seguindo a metodologia de Zhang et al. (2005a,b) e Haylock et al. (2006). O RClimdex fornece 27 índices de extremos climáticos, sendo que neste estudo foram selecionados 22 (Tabela 1) dos quais, 11 para análise de temperatura e 11 para precipitação. Baseado na climatologia da cidade observa-se que os índices de eventos extremos climáticos escolhidos e descritos na Tabela 1 podem ser aplicados para a região, pois seus limiares se encontram condizentes com os valores apresentados na climatologia descrita pelos dados coletados na estação meteorológica estudada. Campina Grande é uma cidade que tem um período frio extenso durante a estação do inverno, na qual as suas temperaturas mínimas diárias são inferiores a $20^{\circ} \mathrm{C}$, e suas temperaturas máximas não ultrapassam os $25{ }^{\circ} \mathrm{C}$. Adicionalmente, a climatologia da cidade não tem frequentes eventos de precipitações diárias superiores a 50 $\mathrm{mm}$. Sendo assim, todos os índices de extremos climáticos adotados nesse estudo representam bem o comportamento da cidade estudada.

Foi elaborada uma análise temporal utilizando o teste de Mann-Kendall para verificar se existe ou não tendência temporal dos índices de extremos climáticos, durante o período de estudo. As tendências temporais (anuais e sazonais) dos índices foram analisadas através do teste não paramétrico de Mann-Kendall (Mann, 1945; Kendall, 1975). A estatística $S$ do teste de MannKendall pode ser calculada de acordo com a Equação 1:

$$
S=\sum_{k=1}^{n-1} \sum_{j=k+1}^{n} \operatorname{sign}\left(x_{j}-x_{k}\right)
$$

O sinal pode ser obtido através das Equações 2, 3 e 4:

$$
\begin{aligned}
& \operatorname{sign}\left(x_{j}-x_{k}\right)=1 \text { se } \quad x_{j}-x_{k}>0 \\
& \operatorname{sign}\left(x_{j}-x_{k}\right)=0 \text { se } x_{j}-x_{k}=0 \\
& \operatorname{sign}\left(x_{j}-x_{k}\right)=-1 \text { se } x_{j}-x_{k}<0
\end{aligned}
$$

A estatística $S$ aproxima-se da normalidade à medida que $n$ cresce, com média e variância definidas, respectivamente, por (Mann, 1945; Kendall, 1975):

$$
\begin{aligned}
& E(\mathrm{~S})=0 \\
& \operatorname{Var}(S)=\frac{n(n-1)(2 n+5)-\sum_{i=1}^{m} t_{i}(i)(i-1)(2 i+5)}{18}
\end{aligned}
$$

$\mathrm{O}$ teste estatístico $\mathrm{Z}(\mathrm{t})$ serve para calcular a tendência de uma série temporal e pode ser obtido de acordo com a Equação 7.

$$
Z(t)=\left\{\begin{array}{r}
\frac{S-1}{\sqrt{\operatorname{Var}(S)}}, \text { se }, S>0 \\
0, \text { se }, S=0 \\
\frac{S+1}{\sqrt{\operatorname{Var}(S)}}, \text { se }, S<0
\end{array}\right.
$$

A hipótese nula de ausência de tendência, $H_{0}$, é rejeitada sempre que $|Z(t)|>Z_{\alpha / 2}$ em que $\alpha$ é o nível de significância adotado e $Z_{\alpha / 2}$ é a variável normal reduzida da função de distribuição normal padrão, para valores $\operatorname{de}(Z(t)>0)$ tendências crescentes, para valores de $(Z(t)<0)$ tendências decrescentes (Santos e Portella, 2008). Os níveis de significância adotados foram de $5 \%(\mathrm{p} \leq 0,05)$ e $1 \%(\mathrm{p} \leq 0,01)$.

A magnitude da tendência foi calculada através do teste não paramétrico de Sen (Sen-Slope) (Sen, 1968), este teste estima a inclinação $\left(T_{i}\right)$ de uma alteração na medição por mudança de tempo, como mostra a Equação 8:

$$
T_{i}=\frac{x_{j}-x_{k}}{j-k}, \text { para } \mathrm{i}=1,2,3, \ldots, \mathrm{N} .
$$


Tabela 1 - Definição dos índices de extremos climáticos dependentes da temperatura e precipitação usados neste estudo.

\begin{tabular}{|c|c|c|c|}
\hline Índices & Nome & Definição & Unidades \\
\hline SU & Dias quentes & $\begin{array}{l}\text { Contagem anual quando } \mathrm{TX} \\
\text { (máximo diário) }>25^{\circ} \mathrm{C}\end{array}$ & dias \\
\hline TR & Noites quentes & $\begin{array}{l}\text { Contagem anual quando } \mathrm{TN} \\
\text { (mínimo diário) }>20^{\circ} \mathrm{C}\end{array}$ & dias \\
\hline TXx & Máx. Tmáx & $\begin{array}{l}\text { Valor máximo mensal da } \\
\text { temperatura máxima diária }\end{array}$ & ${ }^{\circ} \mathrm{C}$ \\
\hline TXn & Mín. Tmáx & $\begin{array}{l}\text { Valor mínimo mensal da } \\
\text { temperatura máxima diária }\end{array}$ & ${ }^{\circ} \mathrm{C}$ \\
\hline $\mathrm{TNx}$ & Máx. Tmín & $\begin{array}{l}\text { Valor máximo mensal da } \\
\text { temperatura mínima diária }\end{array}$ & ${ }^{\circ} \mathrm{C}$ \\
\hline TNn & Mín. Tmín & $\begin{array}{l}\text { Valor mínimo mensal da } \\
\text { temperatura mínimo diária }\end{array}$ & ${ }^{\circ} \mathrm{C}$ \\
\hline TX10p & Dias frios & $\begin{array}{l}\text { Porcentagem de dias quando } \\
\text { TX }<10 \text { percentil }\end{array}$ & dias \\
\hline TX90p & Dias quentes & $\begin{array}{l}\text { Porcentagem de dias quando } \\
\text { TX }>90 \text { percentil }\end{array}$ & dias \\
\hline TN10p & Noites frias & $\begin{array}{l}\text { Porcentagem de dias quando } \\
\text { TN }<10 \text { percentil }\end{array}$ & dias \\
\hline TN90p & Noites quentes & $\begin{array}{l}\text { Porcentagem de dias quando } \\
\text { TN }>90 \text { percentil }\end{array}$ & dias \\
\hline DTR & $\begin{array}{c}\text { Intervalo da temperatura } \\
\text { diurna }\end{array}$ & $\begin{array}{l}\text { Diferença mensal média entre } \\
\text { TX e TN }\end{array}$ & ${ }^{\circ} \mathrm{C}$ \\
\hline RX1day & $\begin{array}{l}\text { Máximo total de } 1 \text { dia de } \\
\text { precipitação }\end{array}$ & $\begin{array}{l}\text { Máximo de precipitação } \\
\text { mensal de } 1 \text { dia }\end{array}$ & $\mathrm{mm}$ \\
\hline RX5day & $\begin{array}{l}\text { Máximo total de } 5 \text { dias } \\
\text { de precipitação }\end{array}$ & $\begin{array}{l}\text { Máximo de precipitação } \\
\text { mensal consecutivo de } 5 \text { dias }\end{array}$ & $\mathrm{mm}$ \\
\hline SDII & $\begin{array}{l}\text { Índice de intensidade } \\
\text { diária simples }\end{array}$ & $\begin{array}{l}\text { Precipitação média anual } \\
\text { quando } P R C P \geq 1,0 \mathrm{~mm}\end{array}$ & $\mathrm{~mm}$ \\
\hline $\mathrm{R} 10 \mathrm{~mm}$ & $\begin{array}{l}\text { Número de dias com } \\
\text { precipitação intensa }\end{array}$ & $\begin{array}{l}\text { Contagem anual de dias em } \\
\text { que } P R C P \geq 10 \mathrm{~mm}\end{array}$ & dias \\
\hline $\mathrm{R} 20 \mathrm{~mm}$ & $\begin{array}{l}\text { Número de dias com } \\
\text { precipitação muito } \\
\text { intensa }\end{array}$ & $\begin{array}{l}\text { Contagem anual de dias em } \\
\text { que } P R C P \geq 20 \mathrm{~mm}\end{array}$ & dias \\
\hline $\mathrm{R} 50 \mathrm{~mm}$ & $\begin{array}{l}\text { Número de dias acima de } \\
50 \mathrm{~mm}\end{array}$ & $\begin{array}{l}\text { Contagem anual de dias em } \\
\text { que } P R C P \geq 50 \mathrm{~mm}\end{array}$ & dias \\
\hline CDD & Dias consecutivos secos & $\begin{array}{l}\text { Número máximo de dias } \\
\text { consecutivos com } R R<1 \mathrm{~mm}\end{array}$ & dias \\
\hline CWD & $\begin{array}{l}\text { Dias consecutivos } \\
\text { úmidos }\end{array}$ & $\begin{array}{l}\text { Número máximo de dias } \\
\text { consecutivos com } R R \geq 1 \mathrm{~mm}\end{array}$ & dias \\
\hline $\mathrm{R} 95 \mathrm{p}$ & Dias muito úmidos & $\begin{array}{l}\text { Precipitação total anual quando } \\
\text { RR }>95 \mathrm{p}\end{array}$ & $\mathrm{mm}$ \\
\hline R99p & $\begin{array}{l}\text { Dias extremamente } \\
\text { úmidos }\end{array}$ & $\begin{array}{l}\text { Precipitação total anual quando } \\
\text { RR >99p }\end{array}$ & $\mathrm{mm}$ \\
\hline PRCPTOT & Precipitação total anual & $\begin{array}{l}\text { Precipitação total anual em dias } \\
\text { úmidos ( } R R \geq 1 \mathrm{~mm})\end{array}$ & $\mathrm{mm}$ \\
\hline
\end{tabular}

onde $T_{i}$ é a inclinação entre os pontos dos dados $x_{j}$ e $x_{k} ; x_{j}$ a medição dos dados em relação ao tempo $j ; x_{k}$ a medição dos dados em relação ao tempo $k$ e $j$ o tempo após o tempo $k$.

A mediana destes $N$ valores de $T_{i}$ é representado como o estimador Sen-Slope, que é dado por (Sen, 1968):

$$
Q_{i}=\left\{\begin{aligned}
\frac{T_{\frac{N+1}{2}},}{2} & N \text { é impar } \\
\frac{1}{2}\left(T_{\frac{N}{2}}+T_{\frac{N+2}{2}}\right), & N \text { é par }
\end{aligned}\right.
$$

O estimador de Sen é descrito como $Q_{\text {med }}=T(N+1) / 2$, se $N$ for par, caso ele seja ímpar é considerado como $Q_{\mathrm{med}}=$
$\left[T_{N / 2}+T_{(N+2) / 2}\right] / 2$. Por fim, $Q_{m e d}$ é calculado por um teste de dois lados em $100(1-\alpha) \%$ de intervalo de confiança e, em seguida, a inclinação pode ser obtida através do teste não paramétrico. Valores positivos de $Q_{i}$ indicam tendência crescente ou ascendente, e um valor negativo de $Q_{i}$ nos dá tendência decrescente ou descendente da série histórica. $\mathrm{O}$ valor encontrado através dos coeficientes de regressão, utilizando métodos quantitativos (métodos dos mínimos quadrados ou o método da máxima verossimilhança), fornece estimativa mais precisa do que o teste $T_{i}$, uma vez que, esses coeficientes baseiam-se em distribuições probabilísticas, conferindo mais 
poder ao se testar hipóteses (Testes Uniformemente Mais Poderosos). Por outro lado, o teste $\mathrm{T}_{i}$ leva em consideração as possíveis correlações ao longo do tempo. O coeficiente de regressão, com seu respectivo $p$-valor, é dado pelo RClimdex.

\section{RESULTADOS E DISCUSSÃO}

O estudo sobre as tendências dos índices de extremos climáticos da cidade de Campina Grande (Tabela 2), correspondente ao período de 1975 a 2011, identificou a magnitude de alguns índices estatisticamente significativos. $\mathrm{Na}$ análise dos vinte e dois índices, observou-se que dessas, oito encontravam-se com nível de significância estatística de $5 \%$, como o SU, TR, TXx, TNx, TX10p, TX90p, TN10p e TN90p. Dentre estes oito índices, em seis o nível de significância estatística foi superior a 1\%, que foram SU, TR, TXx, TX10p, TN10p e TN90p.

Foi observado na Tabela 2, que a frequência em que ocorrem os dias quentes tem aumentado durante as últimas décadas, correspondendo a uma tendência com magnitude de $\mathrm{SU}=0,718$ dias/ano. Esse índice indica aumento no número de dias em um ano em que a temperatura máxima diária foi maior que $25^{\circ} \mathrm{C}$. Não só os dias quentes estão ficando mais frequentes, como também o número de noites quentes. Este índice vem crescendo ao longo dos anos com uma tendência de $\mathrm{TR}=3,127$ dias/ano, ou seja, está ocorrendo aumento no número de dias em um ano em que a temperatura mínima diária é superior a $20^{\circ} \mathrm{C}$. Se a quantidade destes eventos permanecer neste ritmo, o clima da cidade de Campina Grande, que é conhecida no estado da Paraíba por sua característica de clima frio e ameno, se tornará mais aquecido ao longo das próximas décadas, prejudicando o turismo de inverno da região, devido ao aumento do número de dias quentes e provavelmente secos.

A magnitude do valor máximo da temperatura máxima diária (TXx) tem aumentado significativamente cerca de 0,05 ${ }^{\circ} \mathrm{C} /$ ano, assim como também o valor máximo da temperatura mínima diária (TNx), com uma tendência de $0,018{ }^{\circ} \mathrm{C} /$ ano. Devido ao aumento desses dois índices extremos, a amplitude térmica se mantém ao longo de toda a série, não apresentando variações significativas. Assim, observa-se que as temperaturas mínima e máxima têm aumentado gradativamente ao longo das últimas décadas em Campina Grande, o que pode estar relacionado à intensa expansão urbana associada ao aumento do número de ruas com pavimentação asfáltica e redução de áreas com cobertura vegetal.

Conforme visto anteriormente, os valores máximos da temperatura tem aumentado ao longo dos anos, acarretando na diminuição da tendência de ocorrer dias mais frios (TX10p), esta redução, estatisticamente significativa, é de 0,411 dias/ano. A quantidade de dias mais quentes (TX90p) tem aumentado, com uma tendência significativa de 0,395 dias/ano. O número de ocorrência de noites frias (TN10p), decresce, com significância estatística, a uma taxa de 0,474 dias/ano. Similarmente, a tendência de ocorrência de noites quentes (TN90p) está aumentando consideravelmente, isto é, a uma taxa de 0,504 dias/ano.

Devido a alta variabilidade espacial e temporal da precipitação sobre a região estudada, os índices de extremos climáticos dependentes dessa variável, apresentados na Tabela 2, a saber: R10mm, R20mm, R50mm, CDD, CWD, R95p, R99p e PRCPTOT, não mostraram tendências com significância estatística ao nível de 5\%. No entanto, os resultados indicam intensificação dos eventos mais intensos de precipitação, ou seja, os índices RX5day e R50mm mostram tendências positivas, evidenciando que a precipitação total em cinco dias consecutivos e o número de dias no ano com precipitações iguais ou superiores à $50 \mathrm{~mm}$ tendem a aumentar e, além disso, a precipitação total anual (PRCPTOT) também mostra tendência de aumento. É importante observar que o índice dias consecutivos secos (CDD) também aumenta, indicando que esses eventos extremos tendem a ser cada vez mais isolados, o que pode causar sérios danos à sociedade. Isto é, o aumento do número de dias consecutivos secos indica que o período chuvoso está diminuindo, no entanto, a precipitação total anual e os episódios de chuvas intensas (Rx5day e R50mm) mostram tendências de aumento, indicando que os eventos de precipitação estão ocorrendo em intervalos menores de tempo, o que pode trazer danos consideráveis (inundações, deslizamentos, perdas materiais e de vidas) para a população.

De posse das informações obtidas através das análises anuais das tendências dos índices de extremos climáticos estudados, foi necessária a obtenção dos comportamentos sazonais de alguns desses índices, verificando assim, o comportamento das tendências e de seus níveis significativos nas quatro estações do ano. Dos onze índices analisados, apenas o RX5day não apresentou tendência significativa ao nível de $5 \%$, os outros dez índices, de acordo com a estação do ano, apresentaram nível de significância variando entre 5\% e $1 \%$. Vale salientar que esses índices foram escolhidos por fornecer informações mensais e, assim, tornando possíveis as análises sazonais dos mesmos.

Analisando o comportamento sazonal do mínimo valor mensal da temperatura mínima diária (TNn) (Figura 2a), observa-se aumento da temperatura mínima em todas as estações do ano durante o período estudado. Com o auxílio da Tabela 3, constata-se que os valores das magnitudes das tendências foram de $0,043{ }^{\circ} \mathrm{C} / \mathrm{ano}, 0,053{ }^{\circ} \mathrm{C} / \mathrm{ano}, 0,057^{\circ} \mathrm{C} /$ ano e $0,025^{\circ} \mathrm{C} /$ ano para as estações de primavera, verão, outono e inverno, respectivamente. Configuração similar foi observada para o comportamento sazonal do máximo valor mensal da temperatura mínima diária (TNx) (Figura 2b), onde os valores 
Tabela 2 - Tendências dos índices de extremos climáticos para a cidade de Campina Grande - PB, para o período de 1975 a 2011.

\begin{tabular}{|c|c|c|c|c|}
\hline Índices & $\begin{array}{c}\text { Kendall } \\
\text { Tau }\end{array}$ & $\mathbf{Z}$ & p-valor & Tendência \\
\hline SU (dias/ano) & 0,379 & 3,151 & 0,002 & 0,718 \\
\hline TR (dias/ano) & 0,623 & 5,183 & 0,000 & 3,127 \\
\hline $\mathrm{TXx}\left({ }^{\circ} \mathrm{C} / \mathrm{ano}\right)$ & 0,342 & 2,843 & 0,004 & $\mathbf{0 , 0 5 0}$ \\
\hline $\operatorname{TXn}\left({ }^{\circ} \mathrm{C} / \mathrm{ano}\right)$ & 0,118 & 0,981 & 0,326 & 0,020 \\
\hline $\mathrm{TNx}\left({ }^{\circ} \mathrm{C} / \mathrm{ano}\right)$ & 0,234 & 1,942 & 0,052 & 0,018 \\
\hline $\mathrm{TNn}\left({ }^{\circ} \mathrm{C} / \mathrm{ano}\right)$ & 0,069 & 0,575 & 0,565 & 0,011 \\
\hline TX10p (dias/ano) & $-0,544$ & $-4,521$ & 0,000 & $-0,411$ \\
\hline TX90p (dias/ano) & 0,293 & 2,433 & 0,015 & 0,395 \\
\hline TN10p (dias/ano) & $-0,537$ & $-4,462$ & 0,000 & $-0,474$ \\
\hline TN90p (dias/ano) & 0,428 & 3,561 & 0,000 & 0,504 \\
\hline DTR $\left({ }^{\circ} \mathrm{C} / \mathrm{ano}\right)$ & 0,188 & 1,562 & 0,118 & 0,013 \\
\hline RX1day (mm/ano) & $-0,066$ & $-0,549$ & 0,583 & $-0,033$ \\
\hline RX5day (mm/ano) & 0,096 & 0,801 & 0,423 & 0,363 \\
\hline SDII (mm/ano) & 0,056 & 0,465 & 0,642 & 0,025 \\
\hline R10mm (dias/ano) & 0,040 & 0,334 & 0,738 & 0,098 \\
\hline R20mm (dias/ano) & $-0,006$ & $-0,046$ & 0,963 & 0,000 \\
\hline R50mm (dias/ano) & 0,055 & 0,455 & 0,649 & 0,032 \\
\hline CDD (dias/ano) & 0,002 & 0,015 & 0,988 & 0,120 \\
\hline CWD (dias/ano) & $-0,060$ & $-0,502$ & 0,616 & 0,000 \\
\hline R95p (mm/ano) & 0,134 & 1,112 & 0,266 & 1,810 \\
\hline R99p (mm/ano) & 0,055 & 0,46 & 0,645 & 0,000 \\
\hline $\begin{array}{l}\text { PRCPTOT } \\
\text { (mm/ano) }\end{array}$ & 0,144 & 1,201 & 0,230 & 2,379 \\
\hline
\end{tabular}

Valores realçados em cinza são tendências com nível de significância estatística de 5\%;

Valores em negrito realçados em cinza são tendências com nível de significância de $1 \%$.

das magnitudes das tendências foram de $0,043^{\circ} \mathrm{C} /$ ano, $0,050^{\circ} \mathrm{C} /$ ano, $0,051^{\circ} \mathrm{C} /$ ano e $0,037^{\circ} \mathrm{C} /$ ano para as estações de primavera, verão, outono e inverno, respectivamente. Essas tendências ajudam a confirmar a elevação sazonal e, consequentemente, anual da temperatura mínima em Campina Grande, podendo gerar desconforto térmico no futuro. Observando a variabilidade sazonal do mínimo valor mensal da temperatura máxima diária (TXn) (Figura 2c), nota-se tendência de aumento no transcorrer do período e, com o auxílio da Tabela 3, é possível verificar que, em praticamente todas as estações, foram observadas tendências com alta significância estatística, com exceção da estação do verão, quais sejam $0,093{ }^{\circ} \mathrm{C} /$ ano, $0,051{ }^{\circ} \mathrm{C} /$ ano e $0,020^{\circ} \mathrm{C} /$ ano para as estações de primavera, outono e inverno, respectivamente. Os valores sazonais das tendências da temperatura máxima diária (TXx) (Figura 2d), mostram tendências de aumento, assim como no TXn. No entanto, todas as tendências desse índice para as diferentes estações do ano se mostraram estatisticamente significativas ao nível de 5\%, ou seja, $0,075^{\circ} \mathrm{C} /$ ano na primavera, $0,041^{\circ} \mathrm{C} /$ ano no verão, $0,054^{\circ} \mathrm{C} /$ ano no outono e $0,028^{\circ} \mathrm{C} /$ ano no inverno. Logo, o valor máximo da temperatura vem aumentando sazonalmente, como já verificado na média anual analisada na Tabela 2. Como as temperaturas mínimas e máximas apresentaram tendências crescentes ao longo dos anos e as temperaturas mínimas tiveram maior aumento, com exceção da primavera (onde houve aumento de $0,033{ }^{\circ} \mathrm{C} / \mathrm{ano}$ ), a amplitude térmica diária (DTR) (Figura 2e) foi reduzida nas taxas de $0,020^{\circ} \mathrm{C} /$ ano, $0,007^{\circ} \mathrm{C} /$ ano e $0,002{ }^{\circ} \mathrm{C} /$ ano para o verão, outono e inverno, respectivamente.

Avaliando o comportamento sazonal dos dias mais frios (TX10p) (Figura 2f) é observado uma redução da quantidade de dias com temperatura amena durante o ano, obtendo os valores de -0,884 dias/ano na primavera com nível de significância de $1 \%$, de $-0,337$ dias/ano no verão, $-0,332$ dias/ano no outono, chegando ao inverno com um valor de decréscimo em torno de $-0,296$ dias/ano. Analisando a sazonalidade dos dias quentes (TX90p) (Figura 2g), verifica-se que há um aumento com 
Tabela 3 - Tendências sazonais dos índices de extremos climáticos da cidade de Campina Grande - PB, para o período de 1975 a 2011.

\begin{tabular}{lcccc}
\hline \multicolumn{1}{c}{ Índice } & Primavera & Verão & Outono & Inverno \\
\hline $\mathrm{TNn}\left({ }^{\circ} \mathrm{C} / \mathrm{ano}\right)$ & $0,043^{*}$ & $0,053^{* *}$ & $0,057^{* *}$ & $0,025^{*}$ \\
$\mathrm{TNx}\left({ }^{\circ} \mathrm{C} / \mathrm{ano}\right)$ & $0,043^{* *}$ & $0,050^{* *}$ & $0,051^{* *}$ & $0,037^{* *}$ \\
$\mathrm{TXn}\left({ }^{\circ} \mathrm{C} / \mathrm{ano}\right)$ & $0,093^{* *}$ & 0,019 & $0,027^{*}$ & $0,020^{*}$ \\
$\mathrm{TXx}\left({ }^{\circ} \mathrm{C} / \mathrm{ano}\right)$ & $0,075^{* *}$ & $0,041^{* *}$ & $0,054^{* *}$ & $0,028^{*}$ \\
$\mathrm{DTR}\left({ }^{\circ} \mathrm{C} / \mathrm{ano}\right)$ & $0,033^{*}$ & $-0,020$ & $-0,007$ & $-0,002$ \\
$\mathrm{TX} 10 \mathrm{p}($ dias/ano) & $-0,884^{* *}$ & $-0,337$ & $-0,332^{* *}$ & $-0,296^{*}$ \\
$\mathrm{TX} 90 \mathrm{p}($ dias/ano) & $0,621^{* *}$ & $0,450^{* *}$ & $0,621^{*}$ & 0,282 \\
$\mathrm{TN} 10 \mathrm{p}($ dias/ano) & $-0,883^{* *}$ & $-0,594 * *$ & $-0,951^{* *}$ & $-0,828^{* *}$ \\
$\mathrm{TN} 90 \mathrm{p}($ dias/ano) & $0,675^{* *}$ & $0,833^{* *}$ & $0,983^{* *}$ & $0,588^{* *}$ \\
$\begin{array}{l}\mathrm{RX} 1 \text { day } \\
(\mathrm{mm} / \mathrm{ano})\end{array}$ & $-0,335$ & 0,206 & 0,226 & $0,353^{*}$ \\
$\begin{array}{l}\mathrm{RX} 5 \mathrm{day} \\
(\mathrm{mm} / \mathrm{ano})\end{array}$ & $-0,507$ & 0,490 & 0,155 & 0,471 \\
\hline
\end{tabular}

(*) Significativo ao nível de $5 \%(\mathrm{p} \leq 0,05)$

(**) Significativo ao nível de $1 \%(\mathrm{p} \leq 0,01)$

significância estatística entre a primavera e o outono, sendo que no período primavera/verão o nível de significância é de $1 \%$, no período do inverno apesar da tendência informar que está aumentando o número de dias quentes, não há significância estatística. Durante o período da primavera a quantidade de dias quentes tem aumentado 0,621 dias/ano. Já no verão, a magnitude da tendência nos indica um aumento de 0,450 dias/ano e no outono uma tendência de 0,621 dias/ano. Ao comparar estes índices de extremos de temperatura observa-se que há coerência entre eles, ou seja, o número de dias frios é decrescente enquanto que o número de dias quentes tem se mostrado crescente ao longo dos anos.

$\mathrm{Na}$ análise do comportamento sazonal das noites mais frias (TN10p) (Figura 2h), verifica-se uma diminuição com alta significativa estatística durante o período em estudo. $\mathrm{Na}$ estação da primavera a redução foi de $-0,833$ dias/ano, no verão de -0,594 dias/ano, já no outono o decréscimo de noites frias alcançou o valor de quase um dia a menos por ano, pois a magnitude deste índice pode ser observada no valor de $-0,951$ dias/ano, e por fim, no inverno o valor do decréscimo foi de $-0,828$ dias/ano. Analisando-se o número de noites quentes (TN90p) (Figura 2i), com alta significância estatística para todas as estações do ano, identifica-se que na primavera a tendência foi de 0,675 dias/ano, durante o verão de 0,833 dias/ ano de noites mais quentes, no outono de 0,983 dias/ano e no inverno a magnitude do aumento dos dias chegou a 0,588 dias/ ano. Similar ao que ocorreu no período de dias frios e quentes observados, verificou-se que há coerência entre eles, ou seja, o número de dias e noites frios é decrescente enquanto que o número de dias e noites quentes tem se mostrado crescente ao longo dos anos.
Os dados sazonais dos índices de precipitação mostraram tendência com significância estatística apenas para o índice RX1day na estação de inverno (Figura 2j), no qual foi verificado um aumento da máxima precipitação em um único dia da ordem de $0,353 \mathrm{~mm} / \mathrm{dia}$, nas estações de verão e outono também há tendência de aumento, e redução na primavera, no entanto, como já citado, sem significância estatística ao nível de $5 \%$. O índice RX5day (Figura 2k) segue o mesmo padrão característico do RX1day, com tendência negativa na primavera e positiva no verão, outono e inverno, salientando que não há significância estatística para o nível adotado. Esse resultado sugere que devido a primavera ser a estação do ano com as maiores tendências de aumento nas temperaturas máximas, haverá uma dificuldade de ocorrer condensação de vapor de água e, consequentemente, de formação de nuvens sobre a região levando à redução das chuvas em 1 e 5 dias consecutivos. A ausência de tendências com valor significativo para a precipitação pode estar relacionado à sua alta variabilidade temporal e espacial, tendo assim, um alto desvio padrão em torno do valor médio. Sendo assim, é sabido que a Paraíba tem como características climáticas marcantes, as irregularidades, tanto espacial quanto temporal, do seu regime de chuvas. Essas condições climáticas interferem diretamente na produção de alimentos e provocam graves prejuízos (Menezes et al., 2008). Ou seja, no período chuvoso, as chuvas se caracterizam pela forte intensidade, enquanto que no período menos chuvoso, são frequentes as estiagens de duração variável, elevando o risco da exploração agrícola.

Apesar da falta de significância encontrada, segundo o diagnóstico do Atlas Brasileiro de Desastres Naturais (CEPED UFSC, 2011), o município de Campina Grande é o que apresenta maior índice de registros de inundações e enxurradas na Paraíba 


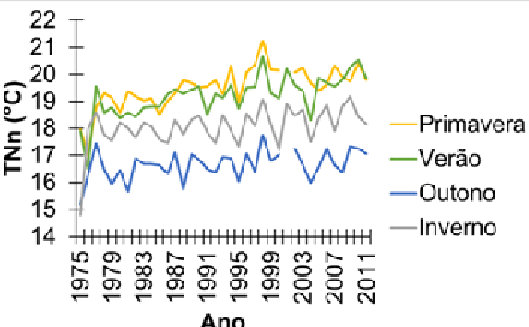

a)

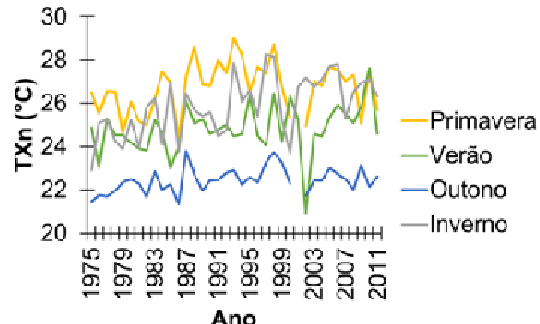

c)

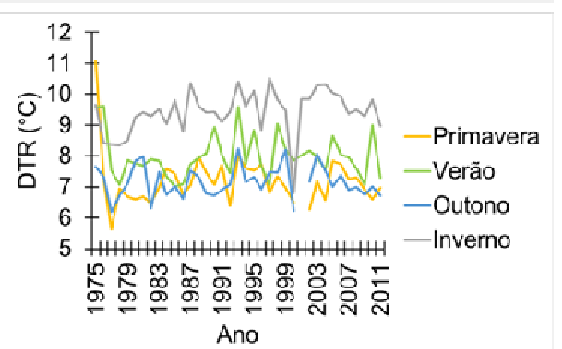

e)

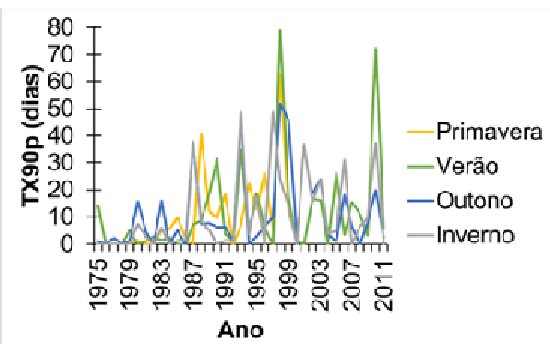

g)

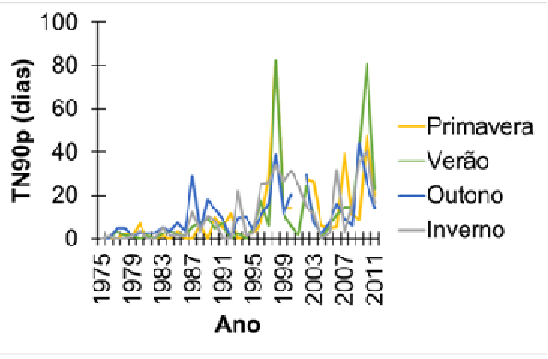

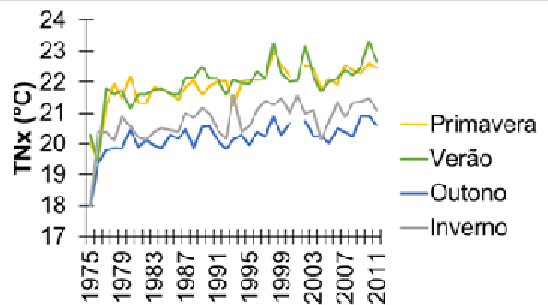

b)

Ano
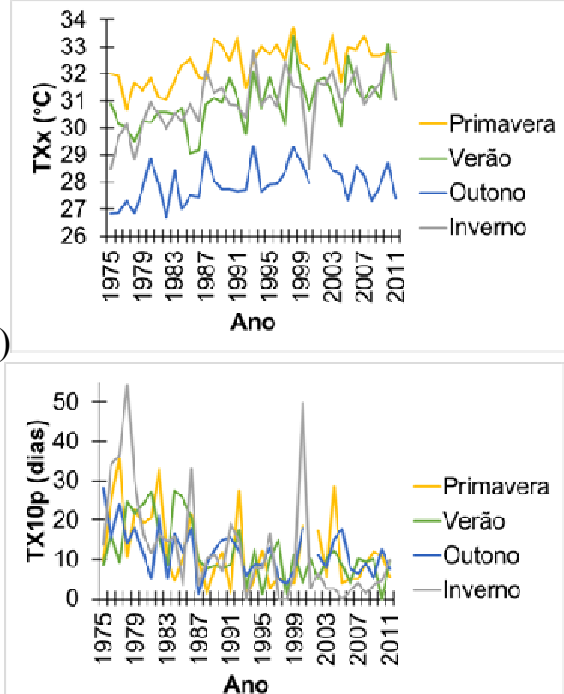

f)

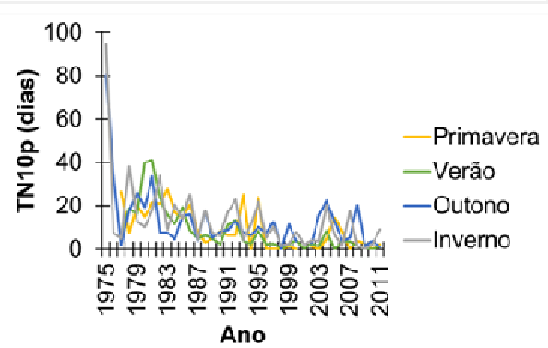

h)

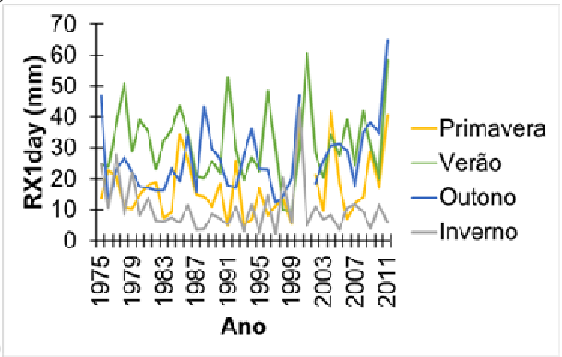

Figura 2 - Variabilidade sazonal dos índices de extremos climáticos estatisticamente significativos para a cidade de Campina Grande - PB, para o período de 1975 a 2011, onde: a) TNn, b) TNx, c) TXn, d) TXx, e) DTR, f) TX10p, g) TX90p, h) TN10p, i) TN90p, j) RX1day e k) RX5day.

desde a década 1990. Vale salientar que os índices extremos de precipitação relacionados a chuvas intensas (RX5day, R50mm e PRCPTOT) apresentaram tendências positivas, mas sem significância estatística. Logo, o aumento no registro de inundações em Campina Grande pode estar associado à mudança da cobertura do solo, ou seja, a impermeabilização do solo. Segundo o mesmo diagnóstico o município é um dos seis do Estado que lideram com o maior número de registros correspondente aos eventos de estiagem e seca (11 ocorrências), o que corrobora com a tendência de 0,120 dias/ano do índice CDD (Tabela 2) e, 4 registros de inundações bruscas e uma ocorrência de inundação gradual. 
O primeiro relatório do Painel Brasileiro de Mudanças Climáticas (PBMC1, 2013), destaca que os cenários climáticos futuros sugerem aumento dos eventos extremos de secas e estiagens prolongadas, principalmente nos biomas da Amazônia, Cerrado e Caatinga, sendo que tais mudanças acentuam-se a partir da metade e final do século XXI, e no que se referem à temperatura do ar na superfície, todas as projeções indicam condições de clima futuro mais quente. Sabe-se que o aumento previsto no PBMC (2013) leva em consideração vários fatores que não estão contemplados diretamente neste estudo, pois o aumento dos índices de temperatura para Campina Grande deve estar diretamente associado aos fatores locais, tais como mudanças na arquitetura da cidade, o que pode alterar circulação de ar na mesma, como também, as expansões das áreas urbanas e das mudanças de uso e cobertura do solo. Essa suposição está fundamentada, por exemplo, no estudo de Chan et al. (2012) que identificaram uma influência da urbanização da ordem de $40 \%$ a $50 \%$ sobre o aquecimento da cidade de Hong Kong nas últimas décadas.

Sabe-se que os eventos extremos climáticos ocorrem em escalas temporais que variam de dias a milênios, no entanto, os mais importantes para as atividades humanas são os extremos de tempo (curto prazo) e os de clima (médio prazo), que são eventos que podem impactar significativamente a sociedade. Nas áreas mais secas, por exemplo, o NEB, conjectura-se que as alterações levem à salinização e desertificação de terras agrícolas, bem como a diminuição da produtividade do gado e das culturas, comprometendo a segurança alimentar da região. Outro impacto marcante é o decréscimo dos recursos hídricos devido ás mudanças climáticas, em consequência do aumento da variabilidade da precipitação com mais veranicos e secas, impactando na recarga das águas subterrâneas. Adicionalmente, o rápido crescimento populacional e consequente demanda hídrica intensificará a vulnerabilidade caso o clima se torne mais quente e seco.

\section{CONCLUSÕES}

A manutenção das estações meteorológicas históricas é muito importante, pois suas longas séries temporais são fundamentais para as análises do clima e suas mudanças. A falta de um banco de dados climáticos de longo prazo tem sido um grande obstáculo para quantificar se os eventos extremos têm mudado nas últimas décadas sobre a Região Nordeste do Brasil. Esse estudo da análise dos extremos de precipitação e temperatura para a cidade de Campina Grande - PB é pioneiro e foi desenvolvido com o objetivo de entender melhor como têm se comportado os eventos extremos de clima na região estudada. Baseado nos resultados observados pode-se concluir que:
1) As temperaturas mínima e máxima diárias estão aumentando gradativamente, com significância estatística, ao longo das últimas décadas;

2) As temperaturas mínimas e máximas diárias apresentaram tendências sazonais crescentes ao longo dos anos e as temperaturas mínimas tiveram maior aumento;

3) O número de dias e noites quentes por ano tem aumentado, com significância estatística, proporcionando assim uma redução significativa dos dias e noites frias;

4) As temperaturas mínimas apresentam maior tendência de aumento na estação do outono, enquanto que as temperaturas máximas mostram maior tendência na primavera;

5) A ausência de tendências com valor significativo para os índices anuais de precipitação está relacionada à irregularidade das chuvas sobre a região Nordeste e, consequentemente, sobre a região estudada, apresentando alta variabilidade espaço-temporal. No entanto, baseado nos índices extremos de precipitação, mesmo sem significância estatística, evidencia-se que as chuvas intensas tendem a aumentar, ocorrendo em um menor intervalo de tempo.

O aumento da temperatura do ar sobre a região contribui com o aumento das taxas de evaporação da água contida no solo e/ou reservatórios, influenciando nas taxas de erosão e umidade do solo e ambas ainda podem ser afetadas por mudanças nos padrões da precipitação. Outro problema que pode se tornar mais frequente sobre a região são as ondas de calor que afetam diretamente na saúde da população, assim como, no consumo energético.

É possível conjecturar que as possíveis causas dessas alterações nos padrões dos índices de extremos climáticos estudados em Campina Grande estão associadas ao adensamento das edificações, modificações no uso do solo nas cercanias da estação meteorológica, como também, às altas taxas de crescimento urbano experimentadas pelas cidades brasileiras de médio a grande porte.

\section{AGRADECIMENTOS}

Os autores agradecem ao Conselho Nacional de Desenvolvimento Científico e Tecnológico (CNPq) pela concessão das bolsas de pós-graduação e Produtividade em Pesquisa para o primeiro e segundo autores, respectivamente, e aos revisores anônimos pelas suas valiosas sugestões e correções.

\section{REFERÊNCIAS BIBLIOGRÁFICAS}

BOCCOLARI, M.; MALMUSI, S.; Changes in temperature and precipitation extremes observed in Modena, Italy. Atmospheric Research, v. 122, p. 16-31, 2013. 
CARNEIRO, I. M. Gerenciamento da demanda de água em áreas verdes públicas: o caso de Campina Grande - PB. (Dissertação de Mestrado do Curso de Pós-Graduação em Engenharia Civil e Ambiental) - Universidade Federal de Campina Grande - UFCG, Campina Grande, 2008.

CEPED UFSC; Atlas brasileiro de desastres naturais 1991 a 2010. V. Paraíba, 2011.

CHAN, H. S.; KOK, M. H.; LEE, T. C.; Temperature trends in Hong Kong from a seasonal perspective. Climate Research, v. 53, p. 53-63, 2012.

CUNHA, J. E. B. L; RUFINO, I. A. A.; IDEIÃO, S. M. A.; Determinação da temperatura de superfície na cidade de Campina Grande-PB a partir de imagens do satélite Landsat 5-TM. Anais XIV Simpósio Brasileiro de Sensoriamento Remoto, Natal, Brasil, INPE, p. 5717-5724, abril de 2009.

DINIZ, J. M. T. Variabilidade da precipitação e do número de dias com chuvas em duas cidades distintas da Paraíba. Holos, v. 3, p. 171-180, 2013.

FOLLAND, C. K.; KARL, T. R.; CHRISTY, J. R.; CLARKE, R. A.; GROUZA, G. V.; JOUZEL, J.; MANN, M. E.; OERLEMANS, J.; SALINGER, M. J.; WANG, S. W. Observed climate variability and change, in Climate Change 2001: The Scientific Basis - Contribution of Working Group I to the Third Assessment Report of the Intergovernmental Panel on Climatic Change, edited by J. T. Houghton et al., pp. 85-97, Cambridge Univ. Press, New York, 2001.

HAYLOCK, M. R.; PETERSON, T. C.; ALVES, L. M.; AMBRIZZI, T.; ANUNCIAÇÃO, T. M. T.; BAEZ, J.; BARROS, V. R.; BERLATO, M. A.; BIDEGAIN, M.; CORONEL, G.; GARCIA, V. J.; GRIMM, A. M.; KAROLY, D.; MARENGO, J. A.; MARINO, M. B.; MONCUNILL, D. F.; NECHET, D.; QUINTANA, J.; REBELLO, E.; RUSTICUCCI, M.; SANTOS, J. L.; TREBEJO, I.; VINCENT, L. A.; Trends in total and extreme South American rainfall 1960-2000 and links with sea surface temperature. Journal of Climate, v. 19, p. 1490-1512, 2006.

INSTITUTO BRASILEIRO DE GEOGRAFIAE ESTATÍSTICA (IBGE).Cidades@. Disponível em http://cidades.ibge.gov. $\mathrm{br} / \mathrm{xtras} /$ perfil.php?lang=\&codmun=250400. Acesso em 24 de janeiro de 2014.

IPCC. 2013. Climate Change 2013 - The Physical Science Basis. Working Group I Contribution to the IPCC Fifth Assessment Report. Summary for Policymakers. IPCC, Stockholm, Sweden.

JONES, P. Global temperature record. Climate Research Unit, University of East Anglia, 2013, http://www.cru.uea.ac.uk/ cru/info/warming/.

KENDALL, M. G. Rank Correlation Methods. London: Charles Griffin, p. 120, 1975.
KOSTOPOULO, E., JONES, P. D. Assessment of climate extremes in the Eastern Mediterranean. Meteorology and Atmospheric Physics, v. 89, p. 69-85, 2005.

LIEBMANN, B.; VERA, C. S.; CARVALHO, L. M. V.; CAMILLONI, I. A.; HOERLING, M. P.; ALLURED, D.; BARROS, V. R.; BÁEZ, J.; BIDEGAIN, M. An observed trend in Central South American precipitation. Journal of Climate, v. 17, p. 4357-4367, 2004.

MANN, H. B. Nonparametric tests against trend. Econometrica, v.13, p.245-259, 1945.

MARENGO, J. A.; JONES, R.; ALVES, L. M.; VALVERDE, M. C.; Future change of temperature and precipitation extremes in South America as derived from the PRECIS regional climate modeling system. Internacional International Journal of Climatology, v. 29, p. 2241-2255, 2009.

MEDEIROS, A. M. T.; SILVA, M. P.; MEDEIROS, R. M.; LIMA, R. C. C.; Mudanças Climáticas em Campina Grande - PB - Um Estudo Sobre o Aquecimento Urbano. Revista Brasileira de Geografia Física, v. 3, n. 2, p. 278 - 285, 2011.

MENEZES, H. E. A.; BRITO, J. I. B.; SANTOS, C. A. C.; SILVA, L. L. A relação entre a temperatura da superfície dos oceanos tropicais e a duração dos veranicos no estado da Paraíba. Revista Brasileira de Meteorologia, v.23, p. 152-161, 2008.

PBMC, 2013: Contribuição do Grupo de Trabalho 1 ao Primeiro Relatório de Avaliação Nacional do Painel Brasileiro de Mudanças Climáticas. Sumário Executivo GT1. PBMC, Rio de Janeiro, Brasil. 24 p.

SANDERSON, M. G.; HEMMING, D. L.; BETTS, R. A. Regional temperature and precipitation changes under highend $\left(>4{ }^{\circ} \mathrm{C}\right)$ global warming. Philosophical Transactions of The Royal Society A, v. 369, p. 85-98, 2011.

SANTOS, C. A. C., BRITO, J. I. B. Análise dos índices de extremos para o semi-árido do Brasil e suas relações com TSM e IVDN. Revista Brasileira de Meteorologia, v. 22, p. 303-312, 2007.

SANTOS, C. A. C.; BRITO, J. I. B.; RAO, T. V. R.; MENEZES, H. E. A. Tendência dos Índices de Precipitação no Estado do Ceará. Revista Brasileira de Meteorologia, v. 24, p. 39-47, 2009.

SANTOS, C. A. C.; MANZI, A. O. Eventos extremos de precipitação no estado do Ceará e suas relações com a temperatura dos Oceanos Tropicais. Revista Brasileira de Meteorologia, v. 26, p. 157-165, 2011.

SANTOS, J. F.; PORTELA, M. M. Quantificação de tendências em séries de precipitação mensal e anual em Portugal Continental, VIII Seminário Ibero Americano sobre Sistemas de Abastecimento Urbano, SEREA 2008, IST, Lisbon, Portugal. 
SEN, P. K. Estimates of the regression coefficient based on Kendall's Tau. Journal of the American Statistical Association, v. 63, p. 1379-1389, 1968.

VINCENT, L. A.; PETERSON, T. C.; BARROS, V. R.; MARINO, M. B.; RUSTICUCCI, M.; CARRASCO, G.; RAMIREZ, E.; ALVES, L. M.; AMBRIZZI, T.; BERLATO, M. A.; GRIMM, A. M.; MARENGO, J. A.; MOLION, L.; MONCUNILL, D. F.; REBELLO, E.; ANUNCIAÇÃO, Y. M. T.; QUINTANA, J.; SANTOS, J. L.; BAEZ, J.; CORONEL. G.; GARCIA, J.; TREBEJO, I.; BIDEGAIN, M.; HAYLOCK, M. R.; KAROLY, D. Observed trends in indices of daily temperature extremes in South America 1960-2000. Journal of Climate, v. 18, p. 5011-5023, 2005. ZHANG, X.; AGUILAR, E.; SENSOY, S.; MELKNYAN, H.; TAGHIYEVA, U.; AHMED, N.; KUTALADZE, N.; RAHIMZADEH, F.; TAGHIPOUR, A.; HANTOSH, T. H.; ALBERT, P.; SEMAWI, M.; ALI, M. K.; HALAL, M.; AL-SHABIBI, A.; AL-OULAN, A.; ZATARI, A.; AL DEAN KHALIL, I.; SAGIR, R.; DEMIRCAN, M.; EKEN, M.; ADIGUZEL, M.; ALEXANDER L,; PETERSON, T. C.; WALLIS, T.; Trends in Middle East climate extremes índices during 1930-2003. Journal of Geophysical ResearchAtmospheres, v. 110, D22104, 2005a.
ZHANG, X.; HEGERL, G.; ZWIERS, F. W.; KENYON, J.; Avoiding inhomogeneity in percentile-based indices of temperature extremes. Journal of Climate, v. 18, p. 16411651, 2005b.

ZHANG, X.; YANG, F.; RClinDex (1.0) User Guide. Climate Research Branch Environment Canada: Downsview, Ontario, Canada, 2004. 